\title{
Decomposition and optimization of linear structures using meta models
}

\author{
Lukas Krischer $^{1}$ (i) . Markus Zimmermann ${ }^{1}$
}

Received: 30 March 2021 / Revised: 29 May 2021 / Accepted: 18 June 2021 / Published online: 9 July 2021

(c) The Author(s) 2021

\begin{abstract}
Monolithic optimization of large mechanical systems can be expensive and cumbersome. Drivers of computational cost and integration effort are, e.g., the size of the design problem and the number of different components, models, and disciplines. Distributed optimization schemes decompose large problems into smaller subproblems; however, they typically require intense coordination effort. This paper proposes an approach for complete decoupling by decomposing a monolithic optimization into independent optimization subproblems that can be solved without need for coordination. This is accomplished by sampling the space of component performance, here represented by eigenvalues and eigenvectors of stiffness matrices, and establishing meta models that map the relevant component performance values onto feasibility and mass estimates. The optimization procedure consists of two steps: First, a system optimization problem is solved by assigning stiffness requirements to components that are approximately feasible and mass-optimal. Second, the component optimization problems are solved independently of each other such that stiffness requirements are satisfied. As information on feasibility and mass is provided during system optimization by meta models, the approach will be referred to as informed decomposition. The effectiveness of the approach is demonstrated by minimizing the mass of a simple two-component linear structure subject to a requirement on total stiffness. This is done for three different component models, a beam with constant cross-section, a beam with varying cross-sections, and an arbitrary 2-dimensional body, using parametric and topology optimization, respectively. The approach produces results that are at most $1 \%$ heavier than the results obtained by monolithic optimization.
\end{abstract}

Keywords Systems design · System optimization · Structural optimization · Topology optimization ·

Top-down development $\cdot$ Meta models

\section{Introduction}

The design of systems with a large number of interacting components can be a difficult task. Two different views are particularly relevant for this paper. First, from a computational performance point of view, the size of the system can make an overall optimization prohibitively expensive demanding a decomposition to reduce the size

Responsible Editor: Junji Kato

Lukas Krischer

lukas.krischer@tum.de

1 Laboratory for Product Development and Lightweight Design, Technical University of Munich, Boltzmannstr. 15, 85748 Garching, Germany of the problem (ElMaraghy et al. 2012). Second, from a designer's perspective, it is often beneficial to think about the entire system without its details. In top-down development processes, requirements are formulated on the system level and then broken down into requirements on components. The components are then typically designed by separate engineering groups (Martins and Lambe 2013; Zimmermann et al. 2017). A decomposition is therefore often preferred over a monolithic optimization utilizing complete system analyses.

In a distributed optimization, the given problem is decomposed into smaller subproblems containing subsets of the objectives, design variables and constraints. However, common approaches as analytical target cascading (Kim et al. 2003), multidisciplinary design optimization of independent subspaces (Shin and Park 2005), or collaborative optimization (Braun et al. 1996) are not fully separable architectures and need a coordination strategy to 
maintain consistency between shared quantities of components and/or the system.

Complete decoupling, i.e., horizontal decomposition between system and component level and vertical decomposition between different components, can avoid further coordination between the components after the decomposition. This kind of quantitative top-down design was introduced by Zimmermann and von Hoessle (2013) and embedded into a design procedure in Zimmermann et al. (2017). Based on system targets, so-called solution spaces are computed representing sets of good, i.e. feasible, designs. The edges of box-shaped solution spaces are permissible intervals for component properties and can be used to formulate completely decoupled component requirements.

Unfortunately, for complete decoupling there is a risk of excluding the optimal, but so far unknown solution beforehand. In Zimmermann and von Hoessle (2013) this is referred to as loss of solution space. One possible way of solving this so-far unsolved problem is to incorporate apriori information from the component level with the help of meta models into the decoupling procedure.

Meta models have a long history in systems design as they can reduce the high computational cost of large size computer models (Viana et al. 2014). For example, deformation energy, referred to as compliance in the context of structural optimization, is often utilized as a performance measure. Since compliance is a load dependent quantity, corresponding mechanical detailed designs are only valid for the respective load case. Kim et al. (2016) carried out a dynamic system optimization with a decomposition of the structure based on a regression model that maps mass on compliance for equivalent static loads of different time steps. Oh et al. (2019) utilized deep learning techniques for estimating topologies with respect to minimal compliance for given normal and shear load cases of a car-wheel. A further generalization to estimate detailed designs with respect to arbitrary load cases is presented in Ulu et al. (2015), Lei et al. (2019), and Yu et al. (2019), where the regression models were trained for multiple load cases. In conclusion, meta models based on compliance need extensive sampling procedures with varying load case conditions to allow for general applicability. Also, compliance does often not accurately represent stiffness related requirements of components as, e.g., maximum displacement. Using compliance here would impose an unnecessary strict constraint to a problem.

Yet, also the displacement of a structure is load dependent. Therefore, quantities are needed that represent inherent component characteristics that are independent of changing boundary conditions as constraints or forces. For linear static analyses, a stiffness matrix incorporates all information about the geometrical and physical constitution of mechanical components and is therefore load-independent. Moreover, it can be directly related to a displacement requirement for a given load case and contains all relevant information regarding the deformation behavior. In Wang et al. (2019), a stiffness matrix-based optimization of a serial robot based on a parametrization of a topology was conducted utilizing a linear regression for the stiffness estimate of the components with respect to mass. In the context of multi-scale optimization, the connection of the macro and micro scale of the structure is sometimes replaced by meta models to reduce the computational cost. In Wu et al. (2019) and Wu et al. (2020) a substructuring technique for hierarchical lattice structures was used to estimate the mass and the stiffness matrix of microstructures. For this purpose, a regression model based on a reduced basis description was established, where the microstructure properties are determined by samples of parametrized unit cells. Furthermore, Wang et al. (2021) carried out a multi-scale topology optimization where meta models of different parameterized microstructures were considered with respect to material properties as stiffness matrix or thermal conductivity. However, due to prescribed parametrization of the geometry, the design freedom in the presented approaches is limited and hence one does not exploit the full lightweight potential. Kollmann et al. (2020) utilized topology optimization to estimate microstructure designs. In order to determine the stiffness matrix, substitute load cases were set up, which were used to optimize different topologies with respect to mass. Utilizing those topologies, a regression model was trained to predict optimal microstructures. However, those meta models do not work directly with a stiffness matrix as an input, but with volume fraction, filter radius, and either maximum bulk modulus, shear modulus, or minimum poison ratio as input variables.

Besides the risk of excluding optimal designs, also ensuring feasibility is a relevant and difficult challenge. In theory, all positive semi-definite stiffness matrices can be realized by a mechanical design. Huang and Schimmels (1998) and Huang and Schimmels (2000) showed that arbitrary spatial stiffness matrices can be realized with a set of so-called screw springs. In practice however, limitations like available geometrical space or the material choice do restrict the feasible design space of stiffness matrices. To avoid requirements on component stiffness that cannot be satisfied, feasibility classification is adopted. In Ding and Vemur (2005), an active learning strategy for feasibility classification of analog circuits was implemented. Jeong et al. (2012) used a support vector machine classifier for mathematical test problems and air-conditioner pipe design. In the context of material design, Jung et al. (2019) modeled feasibility constraints to carry out a material optimization for inverse crystallographic texture problems. For the classification of stiffness matrices, no previous work could be identified. 
This paper presents an approach to decouple system and component optimization of mechanical systems. For this purpose, two sequential optimization steps are proposed. First, a system optimization, where requirements on the elastic behavior of the components, in particular their stiffness matrices, are defined, and the mass and feasibility are estimated by meta models. Thus, the loss of solution space will be reduced. The resulting stiffness matrix requirements are then propagated down to the components. Second, independent component optimization problems are solved. The components are designed via structural optimization techniques, without any coordination with the system level or each other.

The paper is organized as follows: In Section 2, the demonstrator problem setup is explained. The proposed approach is introduced in Section 3. For comparison, in Section 4, two alternative design approaches are shortly described. In Section 5, results for three different component models are presented. The results are discussed in Section 6 and the conclusions presented in Section 7.

\section{Demonstrator problem}

The overall development goal is to design a lightweight mechanical system, consisting of two components, see Fig. 1a, e.g., representing a two-component robot arm. The mechanical structure is clamped on the left side and consists of two components each with length $l_{(i)}=300 \mathrm{~mm}$. The requirement on the system stiffness is:

The system must sustain a vertical load of $F=50 \mathrm{~N}$ with a maximum tip displacement of $d_{0}=1 \mathrm{~mm}$.

(a)

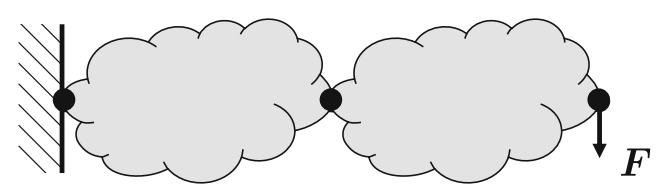

(b)

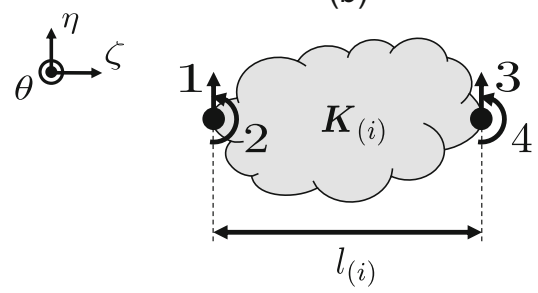

Fig. 1 A simple mechanical two-component system (a), and the respective degrees of freedom, length $l_{(i)}$ and stiffness matrix $\boldsymbol{K}_{(i)}$ for each component $i(\mathrm{~b})$
The payload is applied on the right end of the structure. Each component is modeled as linear elastic and has two mechanical interface points. Each interface possesses two degrees of freedom: one translational and one rotational, see Fig. 1b. The design variable vector $\boldsymbol{x}_{(i)}$ includes all design details for component $i$ on the component-detail level. In the following, three exemplary component models will be considered:

(M1) a beam with constant cross-section,

(M2) a beam with varying cross-sections,

(M3) an arbitrary body represented by a 2-dimensional topology.

For a given material (see Table 1), detailed design variables $\boldsymbol{x}_{(i)}$ determine the component stiffness matrices $\boldsymbol{K}_{(i)} \in \mathbb{R}^{4 \times 4}$ for both components $i$ on the componentperformance level. Under a given load $F$, the structure deforms resulting in a system displacement $\boldsymbol{d}$. Similarly, the detailed description $\boldsymbol{x}_{(i)}$ also defines the mass $m_{(i)}$ of each component and consequently the system mass is $m=$ $m_{(1)}+m_{(2)}$. Figure 2a illustrates the dependencies between all relevant quantities that are needed to solve the given demonstrator problem.

\section{Informed decomposition}

\section{$3.1 \kappa$-representation of the component stiffness matrix $K$}

Not all component stiffness matrices can be realized. Feasible component stiffness matrices $K \in \mathbb{R}^{4 \times 4}$ must satisfy the following requirements:

(R1) $\boldsymbol{K}$ must be symmetric

(R2) rigid body deformations should result in zero forces,

(R3) $\boldsymbol{K}$ must be positive semi-definite, for stability,

(R4) there exists a detail design $\boldsymbol{x}$ corresponding to $\boldsymbol{K}$, that satisfies the problem-specific restriction, e.g., related to geometry.

Requirements (R1)-(R3) shall be satisfied by an appropriate representation of component stiffness matrices $\boldsymbol{K}$ using eigenvectors $\varphi$ of the generalized eigenvalue problem

$K \varphi=\lambda B \varphi$,

Table 1 Material data

\begin{tabular}{llll}
\hline Material & $\begin{array}{l}\text { Young's } \\
\text { Modulus E }(\mathrm{GPa})\end{array}$ & $\begin{array}{l}\text { Poisson's } \\
\text { Ratio } v\end{array}$ & $\begin{array}{l}\text { Density } \rho \\
\left(\mathrm{g} / \mathrm{cm}^{3}\right)\end{array}$ \\
\hline $\mathrm{Al}$ & 70.0 & 0.33 & 2.70 \\
\hline
\end{tabular}


Fig. 2 Dependency graph of all relevant quantities on the system level (I),

component-performance level

(II), and component-detail level

(III) in a monolithic (a) and the proposed approach (b)

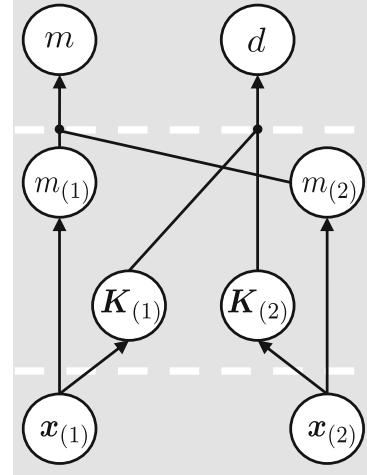

(a)

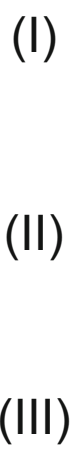

(II)

(III)

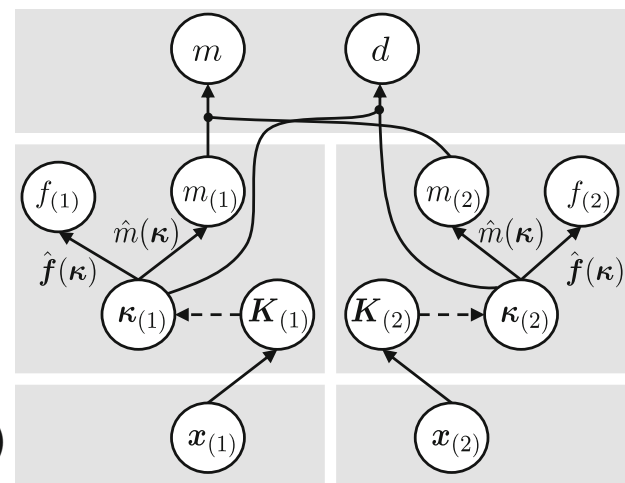

(b) where $\boldsymbol{B} \in \mathbb{R}^{4 \times 4}$ is chosen to adjust the physical units using a reference displacement $d_{r}$, i.e.,

$\boldsymbol{B}=\left[\begin{array}{cccc}1 & 0 & 0 & 0 \\ 0 & d_{r}^{2} & 0 & 0 \\ 0 & 0 & 1 & 0 \\ 0 & 0 & 0 & d_{r}^{2}\end{array}\right]$.

Then, (1) can be rewritten with respect to $\boldsymbol{\Phi}=$ $\left[\boldsymbol{\varphi}_{1}, \ldots, \boldsymbol{\varphi}_{4}\right]$ as $\boldsymbol{K} \boldsymbol{\Phi}=\boldsymbol{B} \boldsymbol{\Phi} \boldsymbol{\Lambda}$, and $\boldsymbol{K}$ can be expressed as

$\boldsymbol{K}=\boldsymbol{B} \boldsymbol{\Phi} \boldsymbol{\Lambda} \boldsymbol{\Phi}^{-1}$,

where $\boldsymbol{\Lambda}=\operatorname{diag}\left(\lambda_{1}, \ldots, \lambda_{4}\right)$. This satisfies (R1). Choosing eigenvectors as rigid body deformations with zero-valued eigenvalues

$\boldsymbol{\varphi}_{1}=\left[\begin{array}{c}d_{r} \\ 0 \\ d_{r} \\ 0\end{array}\right], \quad \lambda_{1}=0$

and

$\boldsymbol{\varphi}_{2}=\left[\begin{array}{r}-d_{r} \\ \frac{2 d_{r}}{l} \\ d_{r} \\ \frac{2 d_{r}}{l}\end{array}\right], \quad \lambda_{2}=0$,

will ensure (R2). (R3) will be satisfied by choosing only eigenvalues equal or greater than zero,

$\lambda_{3} \geq 0, \quad \lambda_{4} \geq 0$.

The remaining eigenvectors $\varphi_{3}$ and $\varphi_{4}$ need to be orthogonal to the already defined $\varphi_{1}$ and $\varphi_{2}$, representing rigid body modes, and each other. The length of the vectors is normalized for uniqueness, i.e.

$\boldsymbol{\varphi}_{m}^{T} \boldsymbol{B} \boldsymbol{\varphi}_{j}= \begin{cases}0 & \text { for } m \neq j \\ d_{r}^{2} & \text { for } m=j\end{cases}$

These equations can be solved to express $\varphi_{3}$ and $\varphi_{4}$ for any given reference displacement $d_{r}$ and component length $l$ in terms of one remaining degree of freedom $\gamma$, denoted as the eigenvector orientation (see Appendix A.1). For $l^{2} \gg$ $d_{r}^{2}$, the expressions can be simplified to

$\boldsymbol{\varphi}_{3}=\left[\begin{array}{c}\frac{d_{r}^{2}}{l}\left(\gamma-\left(1-\gamma^{2}\right)^{\frac{1}{2}}\right) \\ \gamma \\ -\frac{d_{r}^{2}}{l}\left(\gamma-\left(1-\gamma^{2}\right)^{\frac{1}{2}}\right) \\ \frac{-2 \gamma d_{r}^{2}}{l^{2}}-\left(1-\gamma^{2}\right)^{\frac{1}{2}}\end{array}\right]$,

$\boldsymbol{\varphi}_{4}=\left[\begin{array}{c}-\frac{d_{r}^{2}}{l}\left(\gamma+\left(1-\gamma^{2}\right)^{\frac{1}{2}}\right) \\ -\left(1-\gamma^{2}\right)^{\frac{1}{2}} \\ \frac{d_{r}^{2}}{l}\left(\gamma+\left(1-\gamma^{2}\right)^{\frac{1}{2}}\right) \\ \frac{2 d_{r}^{2}}{l^{2}}\left(1-\gamma^{2}\right)^{\frac{1}{2}}-\gamma\end{array}\right]$,

with $0 \leq \gamma<1$. In the following $l=300 \mathrm{~mm}$ and $d_{r}=1$ $\mathrm{mm}$ is set. All component stiffness matrices $\boldsymbol{K}$ satisfying (R1)-(R3) are therefore determined by

$\boldsymbol{\kappa}=\left[\gamma, \lambda_{3}, \lambda_{4}\right]^{T}$.

Satisfying (R4) is less straightforward. Whether or not a component stiffness matrix can be realized will depend on the provided design space and the utilized material. A vector of feasibility estimators $\hat{f}(\boldsymbol{\kappa})=\boldsymbol{f}$ will be established to express feasibility $f_{j}=1$ or any infeasibility $f_{j}=-1$ of a given component stiffness matrix with respect to (R4).

In contrast to the classical problem setup in Fig. 2a, the masses for the $\kappa$-representation of $\boldsymbol{K}$ are not computed by the detailed design variable vector $\boldsymbol{x}$, but with a mass estimator $\hat{m}(\boldsymbol{\kappa})$ for $\boldsymbol{\kappa}$. Thus, a mass estimate is available without knowing the detailed design, see Fig. $2 b$.

Based on the $\kappa$-representation of $\boldsymbol{K}$, the new top-down approach is proposed (see Fig. 3). It is divided into (a) a pure design procedure based on a horizontal and vertical decomposition of a system, enabling a completely independent component design and (b) establishing feasibility estimators $\hat{\boldsymbol{f}}(\boldsymbol{\kappa})$ and mass estimator $\hat{m}(\boldsymbol{\kappa})$, if appropriate models are not available. Note that established meta models can be reused for different design tasks. 


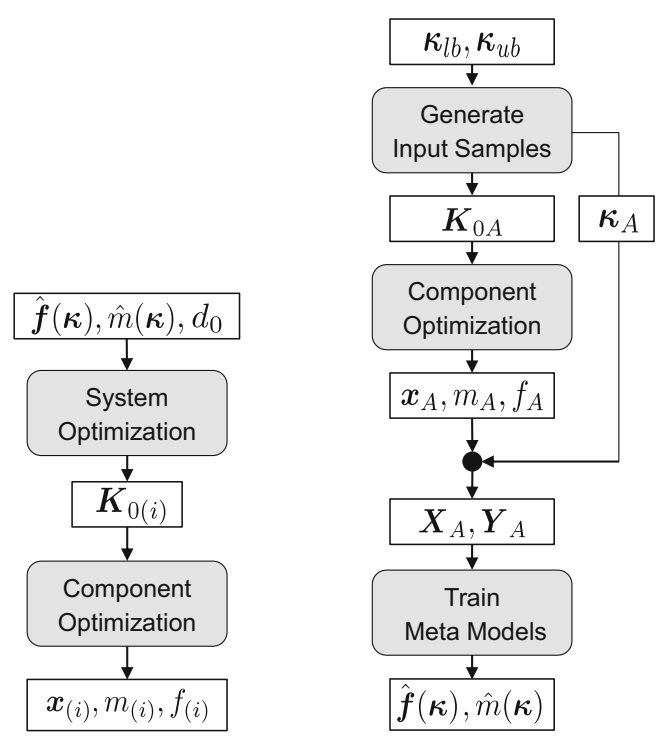

(a)

Fig. 3 Overview over the proposed approach consisting of (a) system optimization and decoupled component optimizations and (b) establishing feasibility estimators $\hat{\boldsymbol{f}}(\boldsymbol{\kappa})$ and mass estimator $\hat{m}(\boldsymbol{\kappa})$

\subsection{System optimization}

For available $\hat{\boldsymbol{f}}(\boldsymbol{\kappa})$ and $\hat{m}(\boldsymbol{\kappa})$, the system optimization decomposes the problem horizontally and vertically into two completely independent component optimizations. The system optimization problem reads:

$$
\begin{array}{cl}
\min _{\boldsymbol{\kappa}_{(1)}, \boldsymbol{\kappa}_{(2)}} \hat{m}_{(1)}\left(\boldsymbol{\kappa}_{(1)}\right) & +\hat{m}_{(2)}\left(\boldsymbol{\kappa}_{(2)}\right) \\
\text { s. t.: } \quad d\left(\boldsymbol{\kappa}_{(1)}, \boldsymbol{\kappa}_{(2)}\right)-d_{0} & \leq 0 \\
-\hat{\boldsymbol{f}}\left(\boldsymbol{\kappa}_{(1)}\right) & \leq 0 \\
-\hat{\boldsymbol{f}}\left(\boldsymbol{\kappa}_{(2)}\right) & \leq 0 \\
\boldsymbol{\kappa}_{l b} \leq \boldsymbol{\kappa}_{(1)} \leq \boldsymbol{\kappa}_{u b} & \\
\boldsymbol{\kappa}_{l b} \leq \boldsymbol{\kappa}_{(2)} \leq \boldsymbol{\kappa}_{u b} . &
\end{array}
$$

The system optimization produces a mass-optimal stiffness $\boldsymbol{\kappa}_{(i)}$ for each component with the help of $\hat{\boldsymbol{f}}(\boldsymbol{\kappa})$ and $\hat{m}(\kappa)$, while not knowing the detailed designs. Note that the system optimization problem between level (I) and (II), see Fig. 2, is significantly less expensive than a monolithic optimization all the way down to level (III) including the detailed design variable vector $\boldsymbol{x}_{(i)}$ for both components. The first requires solving for all component interface displacements, say $\boldsymbol{d}_{s} \in \mathbb{R}^{6}$. This is accomplished by inverting the linear equation system $\boldsymbol{K}_{s} \boldsymbol{d}_{s}=\boldsymbol{F}_{s}$, with $\boldsymbol{K}_{s}\left(\boldsymbol{\kappa}_{(1)}, \boldsymbol{\kappa}_{(2)}\right) \in \mathbb{R}^{6 \times 6}$ being a system stiffness matrix assembled with the two component stiffness matrices $\boldsymbol{K}_{(1)}$ and $\boldsymbol{K}_{(2)}$ represented by $\boldsymbol{\kappa}_{(1)}$ and $\boldsymbol{\kappa}_{(2)}$, respectively, and $\boldsymbol{F}_{S}$ being a vector of loads on component interfaces. By contrast, the second, i.e., monolithic optimization, requires

solving for all nodal displacement of a detailed Finite Element mesh by inverting the linear equation system $\boldsymbol{K}_{s d} \boldsymbol{d}_{s d}=\boldsymbol{F}_{s d}$, with $\boldsymbol{K}_{s d} \in \mathbb{R}^{n_{s d} \times n_{s d}}$ being the detailed system stiffness matrix with $n_{s d}$ degrees of freedom, $\boldsymbol{d}_{s d}$ being the nodal displacement vector, and $\boldsymbol{F}_{s d}$, being the vector of external nodal forces.

A particle swarm algorithm is utilized to solve the given system optimization problem. Note that the algorithm suffers from stochastic perturbations, therefore in the following, $N=10$ optimizations are carried out and the best result is taken.

The resulting $\boldsymbol{\kappa}_{(i)}$ is used to formulate component stiffness target values $\boldsymbol{K}_{0(i)}$ for the two components

$\boldsymbol{K}_{0(i)}=\boldsymbol{B} \boldsymbol{\Phi}_{(i)}\left(\boldsymbol{\kappa}_{(i)}\right) \boldsymbol{\Lambda}_{(i)}\left(\boldsymbol{\kappa}_{(i)}\right) \boldsymbol{\Phi}_{(i)}^{-1}\left(\boldsymbol{\kappa}_{(i)}\right)$.

\subsection{Component optimization}

The component optimization between level (II) and (III), see Fig. 2, identifies the optimal detailed design $\boldsymbol{x}_{(i)}$ for each component with minimum mass for a given reference component stiffness matrix $\boldsymbol{K}_{0(i)}$. The associated problem statement reads

$$
\begin{gathered}
\min _{\boldsymbol{x}_{(i)}} m\left(\boldsymbol{x}_{(i)}\right) \\
\text { s. t.: }\left\|\boldsymbol{K}\left(\boldsymbol{x}_{(i)}\right)-\boldsymbol{K}_{0(i)}\right\| \leq \epsilon \\
\\
g\left(\boldsymbol{x}_{(i)}\right) \quad \leq 0 \\
\boldsymbol{x}_{l b} \leq \boldsymbol{x}_{(i)} \leq \boldsymbol{x}_{u b},
\end{gathered}
$$

where $\boldsymbol{x}_{l b}$ and $\boldsymbol{x}_{u b}$ are the lower and upper bounds on the detailed design variables $\boldsymbol{x}_{(i)}, \boldsymbol{K}\left(\boldsymbol{x}_{(i)}\right)$ is the candidate component stiffness matrix associated with $\boldsymbol{x}_{(i)} \cdot g\left(\boldsymbol{x}_{(i)}\right)$ is an inequality constraint function including additional restrictions for the respective component models.

The optimization problem is solved utilizing the method of moving asymptotes (MMA) by Svanberg (1987). Since the problem was completely decoupled, the system stiffness measured by the total displacement $d$ is assumed to satisfy the requirement $d \leq d_{0}$ as long as the component optimizations are feasible.

\subsection{Establishing feasibility and mass estimator}

When the feasibility estimators $\hat{\boldsymbol{f}}(\boldsymbol{\kappa})$ and the mass estimator $\hat{m}(\boldsymbol{\kappa})$ are not available in closed form solution, meta models are established by first sampling the input space of $\kappa$ satisfying (R1)-(R3), and, second, numerical optimization to assess whether (R4) is satisfied. To create the input samples, first $\kappa_{A}$ is generated within the bounds $\left[\kappa_{l b}, \kappa_{u b}\right]$. The sample vector of the inputs reads

$\boldsymbol{X}_{A}=\left[\kappa_{A}\right]$. 
For each $\boldsymbol{\kappa}_{A}$, a reference component stiffness matrix $\boldsymbol{K}_{0 A}$ is computed using (3). The corresponding detailed design $\boldsymbol{x}$ with minimal mass $m$ is sought using the component optimization of (13). If the component optimization converges, the feasibility flag will be set to $f_{A}=1$ otherwise $f_{A}=-1$. The sample output vector containing the feasibility flag and the realized mass for feasible designs reads

$\boldsymbol{Y}_{A}=\left[f_{A}, m_{A}\right]^{T}$.

The resulting sample data $\left[\boldsymbol{X}_{A}, \boldsymbol{Y}_{A}\right]$ is used to train the meta models $\hat{\boldsymbol{f}}(\boldsymbol{\kappa})$ and $\hat{m}(\boldsymbol{\kappa})$, which can then be utilized for the system optimization of the design procedure of Section 3.2. In Section 5, $\hat{\boldsymbol{f}}(\boldsymbol{\kappa})$ and $\hat{m}(\boldsymbol{\kappa})$ are derived for each given component model.

\section{Alternative design approaches for comparison}

The informed decomposition in Section 3 will be referred to as approach $(A)$ and will be compared to a complete monolithic optimization, which is assumed to be the benchmark result, and an uninformed decomposition, where both components are assigned a certain stiffness budget $\alpha$.

Monolithic optimization, approach (B). This is the standard approach to structural optimization where the mass and the system displacement are directly computed from the detailed design variables $\boldsymbol{x}$ of each component, see Fig. 2a. The associated monolithic optimization problem for approach (B) reads

$$
\begin{array}{ll} 
& \min _{\boldsymbol{x}_{(1)}, \boldsymbol{x}_{(2)}} m\left(\boldsymbol{x}_{(1)}\right)+m\left(\boldsymbol{x}_{(2)}\right) \\
\text { s. t.: } & d\left(\boldsymbol{x}_{(1)}, \boldsymbol{x}_{(2)}\right)-d_{0} \leq 0 \\
& \boldsymbol{g}\left(\boldsymbol{x}_{(1)}, \boldsymbol{x}_{(2)}\right) \leq 0 \\
& \boldsymbol{x}_{l b} \leq \boldsymbol{x}_{(1)} \leq \boldsymbol{x}_{u b} \\
& \boldsymbol{x}_{l b} \leq \boldsymbol{x}_{(2)} \leq \boldsymbol{x}_{u b .} .
\end{array}
$$

Uninformed decomposition, approach $(C)$. This type of vertical decomposition was previously introduced in Krischer et al. (2020) for a similar two-component structure. As an important difference to the proposed approach (A), no mass estimation is used to balance the requirement on the stiffness matrices of each component. Instead, a stiffness distribution is motivated by a reference structure that is not necessarily optimal. Also, no feasibility estimate is taken into account. The total deformation

$d=d_{(1)}+\theta_{(1)} l_{(2)}+d_{(2)}$

can be additively decomposed into the translational displacement $d_{(1)}$ and the rotational displacement $\theta_{(1)}$ of the first component and translational displacement $d_{(2)}$ of the second component (see Fig. 4). The system requirement $d \leq d_{0}$ will be satisfied, whenever the following component requirements are satisfied,

$b_{(1)}(\boldsymbol{x})=\left(d_{(1)}+\theta_{(1)} l_{(2)}\right)-\alpha d_{0} \leq 0$,

$b_{(2)}(\boldsymbol{x})=d_{(2)}-(1-\alpha) d_{0} \leq 0$,

where $\alpha$ can assume any value between 0 and 1 . $\alpha$ controls how much stiffness will be contributed by each component in order to meet the system stiffness requirement. The component optimization of each component reads

$$
\begin{gathered}
\min _{\boldsymbol{x}_{(i)} m\left(\boldsymbol{x}_{(i)}\right)} \\
\text { s. t.: } \quad b\left(\boldsymbol{x}_{(i)}\right) \leq 0 \\
\\
g\left(\boldsymbol{x}_{(i)}\right) \leq 0 \\
\boldsymbol{x}_{l b} \leq \boldsymbol{x}_{(i)} \leq \boldsymbol{x}_{u b} .
\end{gathered}
$$

Two stiffness distributions are chosen for comparison in the following chapters: $\alpha=0.5$ (C.1) and $\alpha=0.6$ (C.2).

\section{Results for three component models}

\subsection{Beam with constant cross-section}

To point out the main features of the proposed approach (A), the simplest component model (M1) containing all necessary ingredients is considered first. For this component model, the feasibility and mass estimators, $\hat{\boldsymbol{f}}(\boldsymbol{\kappa})$ and $\hat{m}(\boldsymbol{\kappa})$, are established. To validate the proposed approach, they are derived in a closed form. A simple mechanical representation with an i-beam and constant cross-sections (see Fig. 5 (M1), for $W(\zeta)=$ const.) is utilized.

The detailed design variables for both components are

$\boldsymbol{x}=\left[\begin{array}{c}w \\ W\end{array}\right] \quad 0<w \leq W \leq 40 \mathrm{~mm}$,

where $w=h$ and $W=H$ are prescribed.
Fig. 4 Additive decomposition of the system displacement $d$ into the respective component displacements $d_{(1)}, \theta_{(1)}$ and $d_{(2)}$
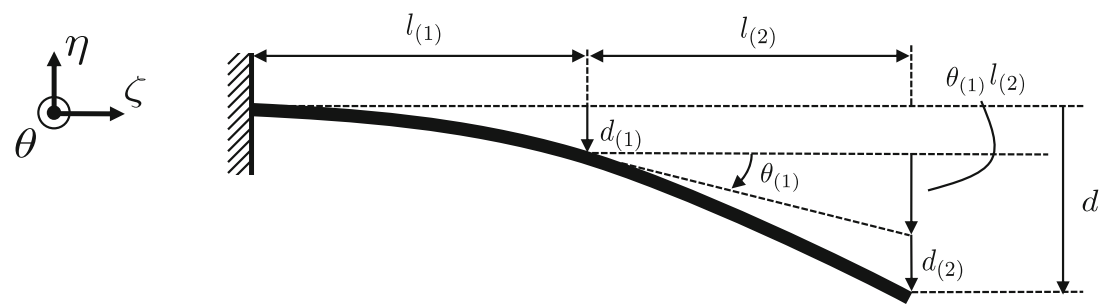


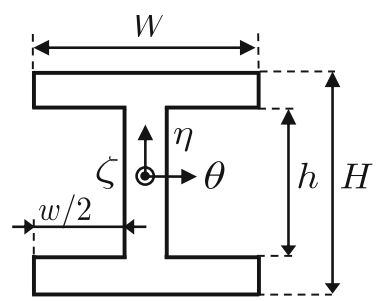

(M1)

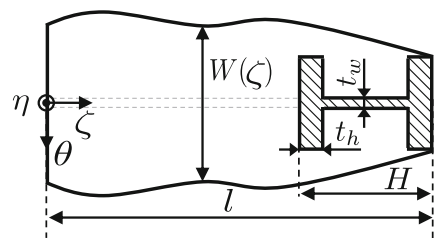

$$
\boldsymbol{x}=\left[W_{e}\right]
$$$$
g(\boldsymbol{x})=[]
$$

(M2)

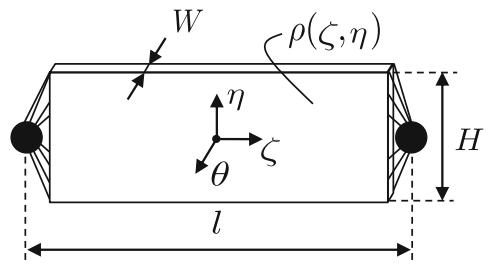

$$
\boldsymbol{x}=\left[\rho_{e}\right]
$$$$
g(\boldsymbol{x})=[]
$$

(M3)

Fig. 5 Design variables of the component models for a beam with constant cross-section (M1), a beam with varying cross-sections (M2), and an arbitrary body represented by a 2-dimensional topology (M3)

The component stiffness matrix $\boldsymbol{K}$ and the mass $m$ for a constant cross-section Euler-Bernoulli beam are according to Bathe (2006)

$$
\begin{aligned}
\boldsymbol{K} & =E I \boldsymbol{k}(l), \\
m & =\rho l(W H-w h),
\end{aligned}
$$

with the moment of inertia $I=1 / 12\left(W H^{3}-w h^{3}\right)$. For physical feasibility, the outer flange width $W$ must be greater than $w$, i.e.,

$g(\boldsymbol{x})=w-W \leq 0$.

The component stiffness matrix $\boldsymbol{K}$ for a given length $l$ and a given material depends solely on the moment of inertia $I$, see (22). Therefore, the stiffness matrix constraint can be simplified to

$\left\|\boldsymbol{K}(\boldsymbol{x})-\boldsymbol{K}_{0}\right\|=I-I_{0}=0$.

Utilizing these simplifications, the solution of the component optimization (13) is

$$
\begin{aligned}
w & =\left(W^{4}-12 I\right)^{\frac{1}{4}}, \\
W & =40 \mathrm{~mm}, \\
m & =\rho l\left(W^{2}-\left(W^{4}-12 I\right)^{\frac{1}{2}}\right),
\end{aligned}
$$

and the sample data can be created as

$$
\begin{aligned}
X_{A} & =\left[\lambda_{3 A}, \lambda_{4 A}, \gamma_{A}\right], \\
\boldsymbol{Y}_{A} & =\left[g_{A}, m_{A}, I_{A}\right]^{T},
\end{aligned}
$$

where the mass sample data is enriched by the inertia information $I_{A}$ to derive the mass estimator with respect to $\kappa$. Since the stiffness only depends on one structural quantity $I$, also the $\kappa$-representation of $\boldsymbol{K}$ can be reduced to one entry. In $\kappa$, the eigenvalues $\lambda_{3}$ and $\lambda_{4}$ represent the stiffness of the beam, which therefore must possess a constant ratio to each other. Consequently, the orientation vector $\gamma$ must be constant. Finally, the upper stiffness bound is needed, which corresponds to a completely filled crosssection. To incorporate all the needed information, only one sample point $N=1$ with the maximum stiffness property is needed. The feasibility estimators $\hat{\boldsymbol{f}}(\boldsymbol{\kappa})$ can then be derived as

$$
\begin{aligned}
& \hat{f}_{1}(\kappa)=\frac{\lambda_{4 A}}{\lambda_{3 A}} \lambda_{3}-\lambda_{4}=c_{1} \lambda_{3}-\lambda_{4}=0, \\
& \hat{f}_{2}(\kappa)=\gamma-\gamma_{A}=\gamma-c_{2}=0, \\
& \hat{f}_{3}(\kappa)=\lambda_{3}-\lambda_{3 A}=\lambda_{3}-c_{3} \leq 0 .
\end{aligned}
$$

The constant ratio is considered in (31). The constant orientation vector $\gamma$ in (32). The upper bound of the first eigenvalue is captured by (33).

For setting up the mass estimator $\hat{m}(\boldsymbol{\kappa})$ with respect to $\lambda_{3}$, the relation between the moment of inertia $I$ and the third eigenvalue is needed $\lambda_{3}$

$I=\frac{\lambda_{3 A}}{I_{A}} \lambda_{3}=c_{4} \lambda_{3}$,

which can be inserted into equation (28)

$\hat{m}(\kappa)=\rho l\left(W^{2}-\left(W^{4}-12 c_{4} \lambda_{3}\right)^{\frac{1}{2}}\right)$.

The established feasibility and mass estimators, $\hat{f}(\boldsymbol{\kappa})$ and $\hat{m}(\boldsymbol{\kappa})$ are shown in Table 2. In Fig. 6, the first row shows the stiffness design space with the accessible space as a one-dimensional line of designs with constant ratio $\lambda_{3}$ and $\lambda_{4}$ and constant eigenvector orientation $\gamma$. Additionally, samples of infeasible designs are shown. Note that most of the design space is infeasible.

Table 2 Explicit formulation of $\hat{\boldsymbol{f}}(\boldsymbol{\kappa})$ and $\hat{m}(\boldsymbol{\kappa})$ for the component model (M1)

\begin{tabular}{ll}
\hline$\hat{\boldsymbol{f}}(\kappa)$ & $\hat{m}(\kappa)$ \\
\hline$\hat{f}_{1}(\kappa)=c_{1} \lambda_{3}-\lambda_{4}=0$, & $\hat{m}(\kappa)=\rho l$ \\
$\hat{f}_{2}\left(\kappa=\gamma-c_{2}=0\right.$, & $\ldots\left(W^{2}-\left(W^{4}-12 c_{4} \lambda_{3}\right)^{\frac{1}{2}}\right)$ \\
$\hat{f}_{3}(\kappa)=\lambda_{3}-c_{3} \leq 0$. & \\
\hline
\end{tabular}



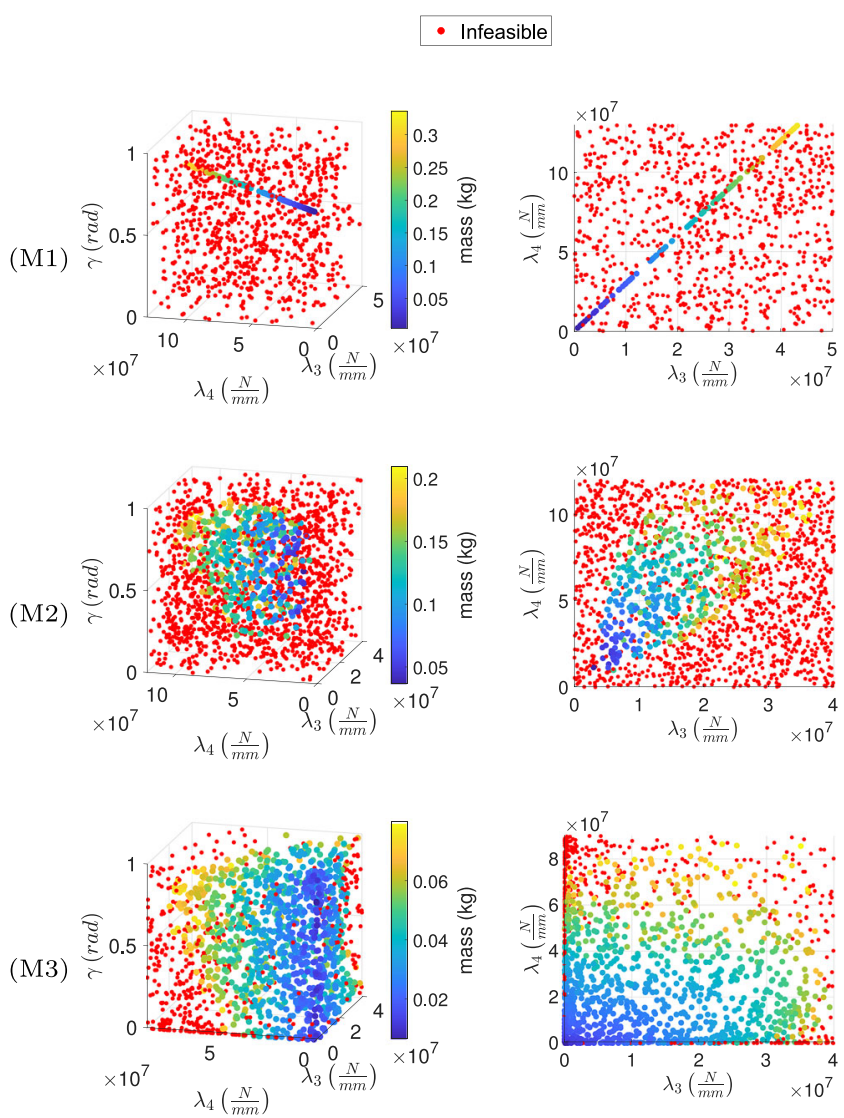

Fig. 6 Sampled stiffness design spaces for the component models (M1)-(M3)

Having set up $\hat{\boldsymbol{f}}(\boldsymbol{\kappa})$ and $\hat{m}(\boldsymbol{\kappa})$, the system optimization (11) can be solved. The results of the system optimization can be seen in the first row of Fig. 7 and in Table 5. The monolithic optimization of approach (B) and the proposed systems design approach (A) have coinciding designs, due to the explicit description of the feasibility and mass estimator. Due to a non-optimal stiffness distribution $\alpha$, the eigenvalues $\lambda_{3}$ and $\lambda_{4}$ of approach (C.1) and (C.2) deviate from the known optimal design, where the eigenvalues of the first component are larger and of the second component lower than in approach (B).

Due to the explicit formulations of $\hat{\boldsymbol{f}}(\boldsymbol{\kappa})$ and $\hat{m}(\boldsymbol{\kappa})$ and (26), (27) and (34), the component-detail design $\boldsymbol{x}$ can be derived directly from the system optimization results $\boldsymbol{\kappa}$. The results of all approaches are shown in Table 6 and Fig. 8. All approaches use the full width $W$ and height $H$, as calculated analytically before. Approaches (A) and (B) show the same cross-sectional description. Consistent with the higher eigenvalues, approach (C) assigns more stiffness to the first component and less to the second component, resulting in a heavier overall structure. In conclusion, all approaches satisfy the given system requirement.
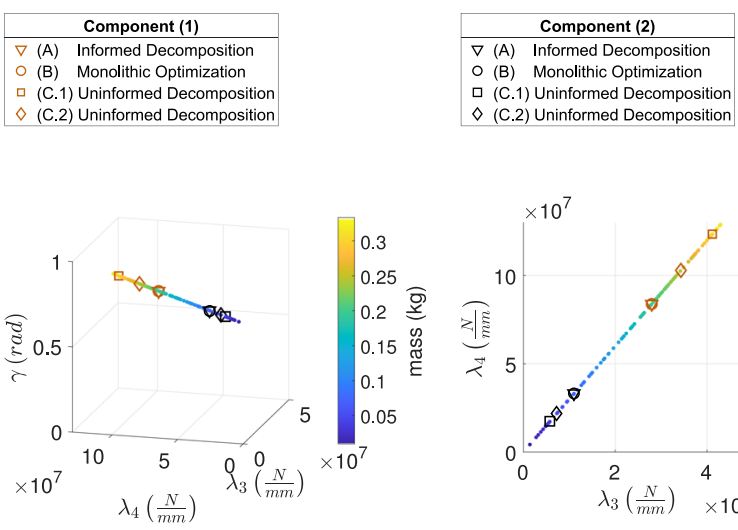

(M1)

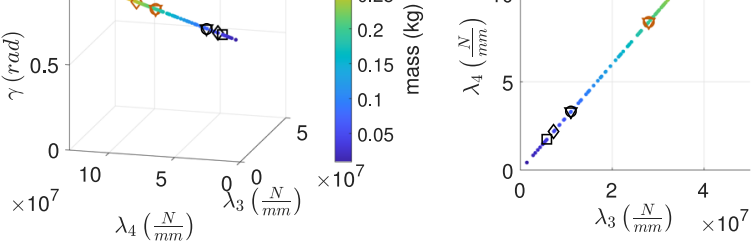

(M2)
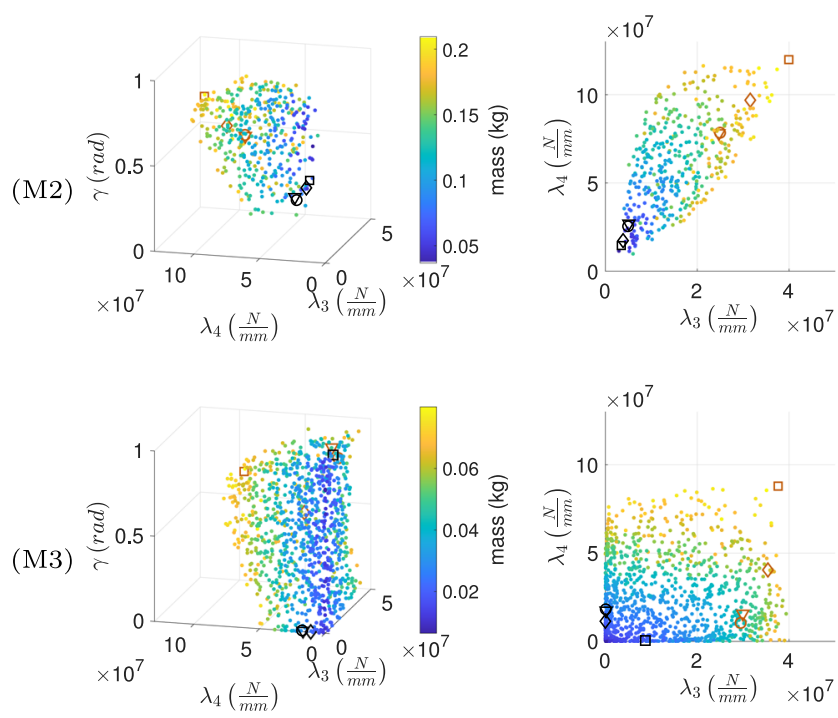

Fig. 7 Feasible and optimized stiffness $\boldsymbol{\kappa}$ for the component models (M1)-(M3)

\subsection{Beam with varying cross-sections}

To increase complexity, varying cross-section widths $W(\zeta)$ are utilized for the second component model (M2). Each structural component is discretized with $n_{e}=10$ crosssections, see Fig. 5 (M2). The detailed design variables for both components are the flange widths $W_{e}$, i.e.,

$$
\begin{aligned}
& \boldsymbol{x}=\left[W_{e}\right] \quad t_{w} \leq W_{e} \leq 40 \mathrm{~mm}, \\
& \text { for } e=1, \ldots, 10,
\end{aligned}
$$

where $H=40 \mathrm{~mm}, h=H-2 t_{h}$, with $t_{h}=3 \mathrm{~mm}$, and $t_{w}=1 \mathrm{~mm}$ are fixed for both components. The width $w$ is calculated as $w=W-t_{w}$. In order to determine the behavior of the components with respect to the defined two interface degrees of freedom, the static Guyan condensation technique is applied to the structure (Guyan 1965), with 

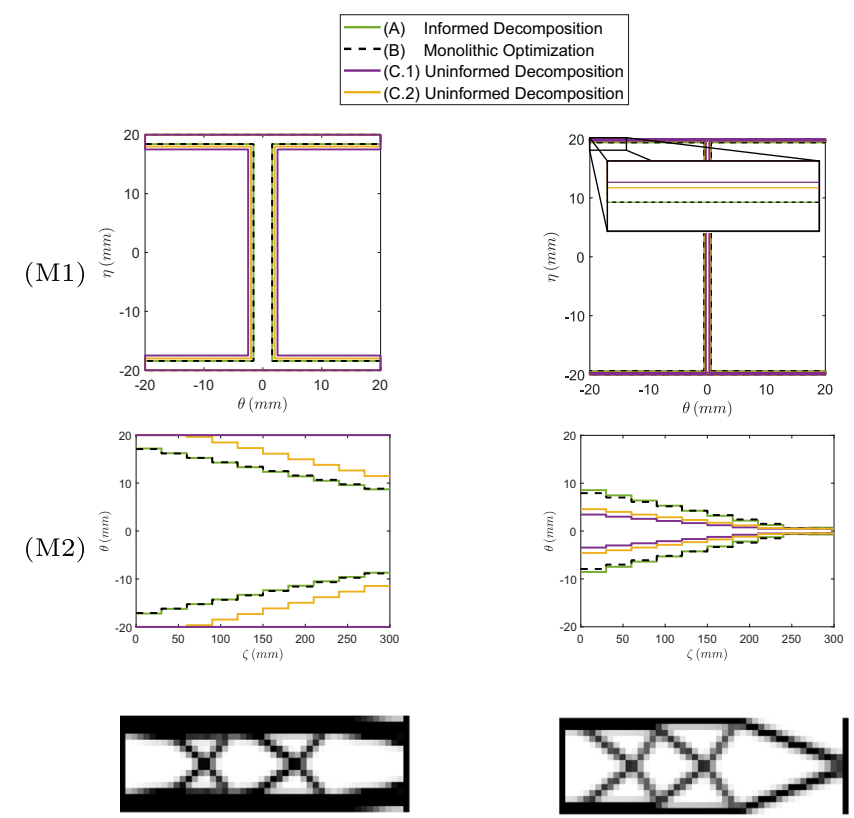

(A)

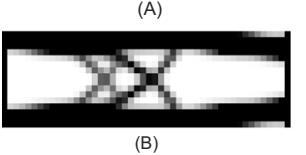

(M3)

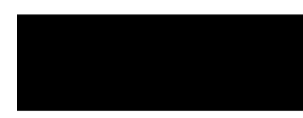

(C.1)

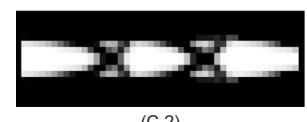

(C. 2)
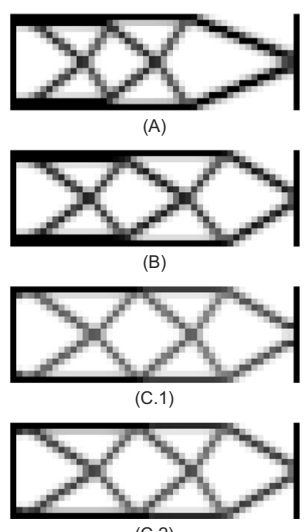

(C. 2$)$

where $\boldsymbol{K}_{g} \in \mathbb{R}^{4 x 4}$ is the condensed interface component stiffness matrix.

The sensitivities of the reduced component stiffness matrix are derived in O'Connell et al. (1976) as

$\frac{\partial \boldsymbol{K}_{g}}{\partial W_{e}}=\boldsymbol{T}_{g}^{T} \frac{\partial \boldsymbol{K}_{d}}{\partial W_{e}} \boldsymbol{T}_{g}$,

where the derivative of the detailed component stiffness matrix $\boldsymbol{K}_{d}$ for $W_{e}$ is

$\frac{\partial \boldsymbol{K}_{d}}{\partial W_{e}}=\frac{H^{3}-h^{3}}{12} E \boldsymbol{k}_{e}\left(l_{e}\right)$.

The mass sensitivities read

$\frac{\partial m}{\partial W_{e}}=\rho l_{e}(H-h)$.

For $\boldsymbol{K}=\boldsymbol{K}_{g}$, the stiffness matrix requirement of the component optimization problem (13) can be reformulated to ensure that requirements (R1)-(R3) are satisfied as (see Appendix A.2)

$-\epsilon \leq\left[\begin{array}{c}\frac{K_{11}-K_{11,0}}{K_{11} .0} \\ \frac{K_{22}-K_{22,0}}{K_{22.0}} \\ \frac{K_{44}-K_{44,0}}{K_{44,0}}\end{array}\right] \leq \epsilon$.

Note that no constraint function $g(x)$ is necessary.

To establish the meta models, $N=1800$ sample points were generated utilizing a Latin hypercube sampling procedure (McKay et al. 1979), see Fig. 6, where $N_{f}=416$ samples were feasible. In contrast to component model (M1), a volume of feasible designs can be observed in the stiffness design space. Due to the lower bound $W_{e} \geq t_{w}$, the feasible stiffness design space has also lower bounds greater than zero $\boldsymbol{\kappa}>\mathbf{0}$. The sample data is used to train a scalarvalued feasibility estimator $\hat{f}(\boldsymbol{\kappa})$ and mass estimator $\hat{m}(\boldsymbol{\kappa})$. Therefore, machine learning methods are applied.

For estimating the feasibility $f$, support vector machines (SVM) are chosen, due to their simplicity combined with convexity also for non-linear classification problems (Cortes and Vapnik 1995). Thus, the binary SVM classifier from MATLAB is utilized and denoted in the following with $\hat{f}_{S V M}(\kappa)$. As a kernel function, radial basis functions are used. The data is split into $80 \%$ training and $20 \%$ test samples. The hyperparameters are determined using a Bayesian optimization with a five-fold cross-validation on the training data. To assess the performance of the classifier, the false positive rate (FP), the true positive rate (TP) and the accuracy (acc) were analyzed, see Table 3. Since a decision for an infeasible design on the system level, makes the whole design approach invalid, the most important quantity is a low FP. Secondly, the mass estimator has to be established using a regression analysis. Feedforward artificial neural networks (ANN), with their capacity of approximating any function with any desired accuracy for a suitable parameter choice, have

$$
\begin{aligned}
& \boldsymbol{K}_{g} \boldsymbol{d}_{m}=\boldsymbol{F}_{m}, \\
& \boldsymbol{K}_{g}=\boldsymbol{T}_{g}^{T} \boldsymbol{K}_{d} \boldsymbol{T}_{g}, \\
& \boldsymbol{T}_{g}=\left[\begin{array}{c}
\mathbf{1} \\
-\boldsymbol{K}_{s s}^{-1} \boldsymbol{K}_{s m}
\end{array}\right],
\end{aligned}
$$


been frequently applied to a wide variety of regression problems (Hornik et al. 1989; Bishop 2009). Hence, the mass estimator is realized with an ANN $\hat{m}_{A N N}(\boldsymbol{\kappa})$ trained with the Levenberg-Marquardt algorithm in MATLAB. The sample data is split into $80 \%$ training, $10 \%$ validation and $10 \%$ test samples. For choosing the best configuration of hidden layers and respective neurons, a grid search was carried out for $n_{h l}=1, \ldots, 5$ hidden layers and $n_{n}=1, \ldots, 10$ neurons. The configuration with the lowest $R^{2}$-value was chosen. The final ANN possesses $n_{h l}=2$ hidden layers and $n_{n}=7$ neurons. Additionally, the mean squared error (mse) of the mass was taken as a second performance measure, see Table 3. Note that the meta models are valid for both components.

Having established the meta models, the system optimization (11) is carried out. The results of the system optimization can be seen in in Fig. 7 and in Table 5. Approaches (A) and (B) again produce similar system designs for both components. In contrast, the uninformed approaches (C.1) and (C.2) show higher and lower eigenvalues for the first and second component, respectively. However, now not only the eigenvalues $\lambda_{3}$ and $\lambda_{4}$, but also the eigenvector orientation $\gamma$ differs.

The results of the subsequent component optimization and the corresponding alternative approaches are shown in Fig. 8 and in Table 6 . The width $W$ decreases along the $\zeta$-axis of both components, as it would be expected. The lightest designs are achieved by approaches (A) and (B), which slightly differ in the weight of the components and the overall mass. Both approaches (A) and (B) satisfy the system requirement. Note that approach (C.1) is not capable of fulfilling the component requirement of the first component and hence also the system requirement is violated resulting in a constant full width $W_{e}$ of component one. In contrast, approach (C.2) satisfies the system requirement, however resulting in a higher total weight compared to (A) and (B).

\subsection{2-dimensional topology}

Finally, arbitrary 2-dimensional bodies are designed utilizing topology optimization (M3). Both sides of each body are connected rigidly to interface points. The height is set

Table 3 Performance measures of $\hat{f}_{S V M}(\boldsymbol{\kappa})$ and $\hat{m}_{A N N}(\boldsymbol{\kappa})$ for the component model (M2)

\begin{tabular}{|c|c|c|c|c|}
\hline \multicolumn{3}{|c|}{$\hat{f}_{S V M}(\boldsymbol{\kappa})$} & \multicolumn{2}{|c|}{$\hat{m}_{A N N}(\boldsymbol{\kappa})$} \\
\hline FP & TP & $\mathrm{acc}$ & $R^{2}$ & $\mathrm{mse}\left(\mathrm{kg}^{2}\right)$ \\
\hline 0.00 & 1 & 1 & 0.996 & $4.42 e-3$ \\
\hline
\end{tabular}

to $H=98 \mathrm{~mm}$ and the thickness to $W=1 \mathrm{~mm}$ for both components, see Fig. 5 (M3).

The body is discretized with uniform 2d-plane stress elements with stiffness matrices $\boldsymbol{K}_{e}$ (Bathe 2006) and $n_{\zeta}=48$ elements in the $\zeta$ - and $n_{\eta}=16$ elements in the $\eta$ direction. The total number of elements is $n_{e}=768$ and the respective element lengths are $l_{e}=\frac{0.98 l}{n_{e \zeta}}$, leaving $1 \%$ of the length for the connection between the body and the interface points on both sides, $H_{e}=\frac{H}{n_{e \eta}}$, and $W_{e}=W$. For the component optimization, the classical SIMP approach (Bendsøe and Sigmund 2004) is used with the element densities $\rho_{e}$ as the detailed design variables, a penalty factor of $p=3$ and a sensitivity filter radius of $r=1.2$ :

$$
\begin{aligned}
& \boldsymbol{K}_{d}=\sum_{e=1}^{n_{e}} \rho_{e}^{p} \boldsymbol{K}_{e}, \\
& \boldsymbol{x}=\left[\rho_{e}\right], \quad 0<\rho_{e} \leq 1 .
\end{aligned}
$$

The optimization code is based on Sigmund (2001). First, the known static condensation technique (Guyan 1965) is applied, with respect to the boundary degrees of freedom of both, the left and the right side of the plate, as the master nodes

$\boldsymbol{K}_{g}=\boldsymbol{T}_{g}^{T} \boldsymbol{K}_{d} \boldsymbol{T}_{g}$.

In order to connect both sides of the body to the interface points, master-slave elimination via a multifreedom constraint is applied, see Liu and Quek (2013). The slave nodes of both sides are eliminated enforcing a geometrical constraint with respect to the master interface nodes yielding

$\boldsymbol{K}_{r g}=\boldsymbol{T}_{r}^{T} \boldsymbol{K}_{g} \boldsymbol{T}_{r}$.

The derivatives of the component stiffness matrix $\boldsymbol{K}_{r g} \in$ $\mathbb{R}^{4 \times 4}$ are

$$
\begin{aligned}
\frac{\partial \boldsymbol{K}_{r g}}{\partial \rho_{e}} & =\boldsymbol{T}_{r}^{T}\left(\boldsymbol{T}_{g}^{T} \frac{\partial \boldsymbol{K}_{d}}{\partial \rho_{e}} \boldsymbol{T}_{g}\right) \boldsymbol{T}_{r}, \\
\frac{\partial \boldsymbol{K}_{d}}{\partial \rho_{e}} & =p \rho_{e}^{p-1} \boldsymbol{K}_{e},
\end{aligned}
$$

and the mass gradients are constant

$\frac{\partial m}{\partial \rho_{e}}=\rho l_{e} H_{e} W_{e}$,

with $\rho$ as the density of aluminum.

Having computed the derivatives, the component optimization problem (13) can be set up utilizing the reduced stiffness constraint of Section 5.2. Again, no extra constraint $g(\boldsymbol{x})$ is required.

The component optimization problem is then used to establish the meta models. While sampling, it could be observed that for low-valued eigenvalues $\lambda_{3}$ and $\lambda_{4}$ the designs tend to be infeasible. Also, the component mass $m$ exhibited a more nonlinear behavior for both eigenvalues 
Table 4 Performance measures of $\hat{f}_{S V M}(\boldsymbol{\kappa})$ and $\hat{m}_{A N N}(\boldsymbol{\kappa})$ for the component model (M3)

\begin{tabular}{|c|c|c|c|c|}
\hline \multicolumn{3}{|c|}{$\hat{f}_{S V M}(\boldsymbol{\kappa})$} & \multicolumn{2}{|c|}{$\hat{m}_{A N N}(\boldsymbol{\kappa})$} \\
\hline FP & $\mathrm{TP}$ & acc & $R^{2}$ & mse $\left(\mathrm{kg}^{2}\right)$ \\
\hline 0.0127 & 0.951 & 0.962 & 0.997 & $9.91 e-4$ \\
\hline
\end{tabular}

in the low-value region. Therefore, the basic sample data $\boldsymbol{X}_{\text {lhs }} \in[0,1]$ created by Latin hypercube sampling is mapped onto a cubic space $\boldsymbol{X}_{l h s} \rightarrow \boldsymbol{X}_{l h s}^{3}$ for both eigenvalues $\lambda_{3}$ and $\lambda_{4}$ and is then applied to the stiffness design space $\boldsymbol{\kappa} \in\left[\boldsymbol{\kappa}_{l b}, \boldsymbol{\kappa}_{u b}\right]$ allowing for more sample data in the crucial low-value region, see Fig. 6. The increased design freedom of the topology optimization provides a broad feasible design space with $N_{f}=1026$ feasible sample points.

The created sample data is used to train the meta models $\hat{f}_{S V M}(\boldsymbol{\kappa})$ and $\hat{m}_{A N N}(\boldsymbol{\kappa})$ similar to Section 5.2. The ANN has $n_{h l}=3$ hidden layers and $n_{n}=9$ neurons. The training results are shown in Table 4.

Next, the system optimization is carried out. The results are shown in Fig. 7 and Table 5. The designs of the first component of approaches (A) and (B) differ slightly with respect to both eigenvalues $\lambda_{3}, \lambda_{4}$ and the eigenvector

Table 5 Optimized stiffness $\boldsymbol{\kappa}$ for the component models (M1)-(M3)

Component modelApproach

\begin{tabular}{|c|c|c|c|c|c|}
\hline \multirow[t]{2}{*}{ (M1) } & & (A) & (B) & (C.1) & (C.2) \\
\hline & $\operatorname{rad})$ & 0.707 & 0.707 & 0.707 & 0.707 \\
\hline \multirow[t]{3}{*}{$\boldsymbol{\kappa}_{(1)}$} & $\frac{\mathrm{N}}{\mathrm{mm}}$ & $2.79 e 7$ & $2.79 e 7$ & $4.11 e 7$ & $3.43 e 7$ \\
\hline & $\frac{\mathrm{N}}{\mathrm{mm}}$ & $8.38 e 7$ & $8.38 e 7$ & $1.23 e 8$ & $1.03 e 8$ \\
\hline & $\mathrm{rad}$ & 0.707 & 0.707 & 0.707 & 0.707 \\
\hline \multirow[t]{2}{*}{$\boldsymbol{\kappa}_{(2)}$} & $\frac{\mathrm{N}}{\mathrm{mm}}$ & $1.11 e 7$ & $1.11 e 7$ & $5.88 e 6$ & $7.35 e 6$ \\
\hline & $\frac{\mathrm{N}}{\mathrm{mm}}$ & $3.34 e 7$ & $3.34 e 7$ & $1.76 e 7$ & $2.20 e 7$ \\
\hline \multirow[t]{2}{*}{ (M2) } & & (A) & (B) & (C.1) & (C.2) \\
\hline & $\operatorname{rad})$ & {$[0.576$} & {$[0.581$} & {$[0.7071]$} & {$[0.5904]$} \\
\hline \multirow[t]{3}{*}{$\kappa_{(1)}$} & $\frac{\mathrm{N}}{\mathrm{mm}}$ & $2.47 e 7$ & $2.50 e 7$ & $3.99 e 7$ & $3.15 e 7$ \\
\hline & $\left.\frac{\mathrm{N}}{\mathrm{mm}}\right)$ & $7.81 e 7$ & $7.85 e 7$ & $1.20 e 8$ & $9.69 e 7$ \\
\hline & $\mathrm{rad})$ & 0.345 & 0.329 & 0.4582 & $0.4107^{7}$ \\
\hline \multirow[t]{2}{*}{$\boldsymbol{\kappa}_{(2)}$} & $\frac{\mathrm{N}}{\mathrm{mm}}$ & $5.22 e 6$ & $5.05 e 6$ & $3.58 e 6$ & $3.97 e 6$ \\
\hline & $\left.\frac{\mathrm{N}}{\mathrm{mm}}\right)$ & $2.69 e 7$ & $2.54 e 7$ & $1.44 e 7$ & $1.72 e 7$ \\
\hline \multirow[t]{2}{*}{ (M3) } & & (A) & (B) & (C.1) & (C.2) \\
\hline & $\operatorname{rad})$ & {$[0.926$} & {$[0.870$} & {$[0.707$} & {$[0.503$} \\
\hline \multirow[t]{3}{*}{$\boldsymbol{\kappa}_{(1)}$} & $\frac{\mathrm{N}}{\mathrm{mm}}$ & $2.99 e 7$ & $2.95 e 7$ & $3.76 e 07$ & $3.54 e 7$ \\
\hline & $\frac{\mathrm{N}}{\mathrm{mm}}$ & $1.57 e 7$ & $1.05 e 7$ & $8.79 e 7$ & $4.05 e 7$ \\
\hline & $\mathrm{rad}$ & $3.67 e-3$ & $2.40 e-3$ & 0.999 & $1.70 e-3$ \\
\hline \multirow[t]{2}{*}{$\boldsymbol{\kappa}_{(2)}$} & $\frac{\mathrm{N}}{\mathrm{mm}}$ & $2.60 e 5$ & $1.81 e 5$ & $8.83 e 6$ & $1.07 e 5$ \\
\hline & $\left.\frac{\mathrm{N}}{\mathrm{mm}}\right)$ & $1.70 e 7$ & $1.78 e 7$ & $8.05 e 4$ & $1.10 e 7$ \\
\hline
\end{tabular}

orientation $\gamma$. The approaches (C.1) and (C.2) assign again more stiffness, i.e. larger eigenvalues, to the first component than to the second one. It is also noticeable that the second component of approach (C.1) produces an eigenvector orientation $\gamma$ close to the upper bound with a larger third eigenvalue $\lambda_{3}$ and a smaller fourth eigenvalue $\lambda_{4}$, in contrast to the other approaches with an eigenvector orientation close to the lower bound and a higher $\lambda_{4}$ eigenvalue compared to $\lambda_{3}$.

The final topologies of the component optimization are shown in Fig. 8. For all design models, the second components converge to the known cantilever beam design for classical bending load cases. The proposed approach (A) exhibits a more tapered right end compared to (B), whereas (C.1) and (C.2) only differ in the assigned densities of the existing beam-like structure resulting in lower component masses (see Table 6). Even though the results of the system optimization of component one are different for (C.1) compared to (A), (B), and (C.2), the results of the component topology optimization in Fig. 8 show only slight differences. The first component of approaches (A) and (B) show thick beam-like structures at the outer regions of the design domain, with crossbars for transferring the shear loads. Yet, the position for these differs in both approaches. Approach (C.2) produces a similar topology, with a slightly higher material fraction in component one. In contrast, the domain of approach (C.1) is completely filled with material, stemming from a component requirement that is too challenging. The required system displacement for approach (C.1) is therefore violated, while approaches (A), (B) and (C.2) are all feasible.

Table 6 Optimized component designs $\boldsymbol{x}$ of the component models (M1)-(M3)

\begin{tabular}{llllll}
\hline Component model & \multicolumn{5}{l}{ Approach } \\
\hline$(\mathrm{M} 1)$ & & $(\mathrm{A})$ & $(\mathrm{B})$ & $(\mathrm{C} .1)$ & $(\mathrm{C} .2)$ \\
$m_{(1)}$ & $(\mathrm{kg})$ & 0.196 & 0.196 & 0.303 & 0.246 \\
$m_{(2)}$ & $(\mathrm{kg})$ & 0.075 & 0.075 & 0.039 & 0.049 \\
$m$ & $(\mathrm{~kg})$ & 0.272 & 0.272 & 0.342 & 0.295 \\
$d$ & $(\mathrm{~mm})$ & 1.00 & 1.00 & 1.00 & 1.00 \\
$(\mathrm{M} 2)$ & & $(\mathrm{A})$ & $(\mathrm{B})$ & $(\mathrm{C} .1)$ & $(\mathrm{C} .2)$ \\
$m_{(1)}$ & $(\mathrm{kg})$ & 0.1527 & 0.1536 & 0.1873 & 0.2219 \\
$m_{(2)}$ & $(\mathrm{kg})$ & 0.0664 & 0.0653 & 0.0434 & 0.0487 \\
$m$ & $(\mathrm{~kg})$ & 0.2191 & 0.2189 & 0.2307 & 0.2707 \\
$d$ & $(\mathrm{~mm})$ & 1.00 & 1.00 & 1.02 & 1.00 \\
$(\mathrm{M} 3)$ & & $(\mathrm{A})$ & $(\mathrm{B})$ & $(\mathrm{C} .1)$ & $(\mathrm{C} .2)$ \\
$m_{(1)}$ & $(\mathrm{kg})$ & 0.0454 & 0.0446 & 0.0810 & 0.0611 \\
$m_{(2)}$ & $(\mathrm{kg})$ & 0.0276 & 0.0282 & 0.0208 & 0.0228 \\
$m$ & $(\mathrm{~kg})$ & 0.0730 & 0.0728 & 0.1018 & 0.0839 \\
$d$ & $(\mathrm{~mm})$ & 1.00 & 1.00 & 1.16 & 1.00 \\
\hline & & & & &
\end{tabular}




\section{Discussion}

Validity The proposed approach (A) was capable of successfully solving the given demonstrator problem for three different component models (M1)-(M3) (see Fig. 9). For the component model (M1), the exact expression for the feasibility and the mass estimator led to the same component-detail designs as for the monolithic system optimization of approach (B). For the component models (M2) and (M3), approach (A) utilized machine learningbased meta models. Here, the system mass $m$ only slightly differed with a deviation below $1 \%$.

Informed vs. uninformed decomposition The key ingredients, the feasibility and the mass estimators, proofed to be crucial within the scope of completely decoupled optimization schemes. The uninformed approach $(\mathrm{C})$ turned out to be both, at risk of proposing infeasible designs (C.1) and producing no mass-optimal solutions (C.2). For more complex design tasks with multiple load cases, the intuition for reasonable component requirements will eventually fail. Therefore, the proposed approach presents a promising way of decomposing multi-component systems in a feasible and mass-optimal way.

Computational cost One motivation of decomposing multicomponent systems is the high computational cost. For the given problem, the computational cost of a monolithic system optimization is still small compared to the sampling process, where each sample point needs to be generated by carrying out a single component optimization. However,
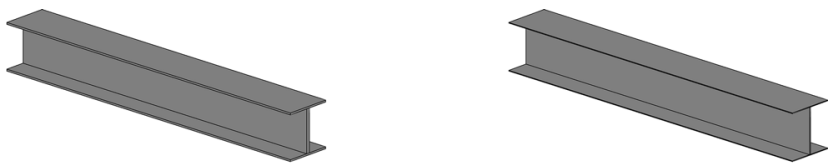

(M1)
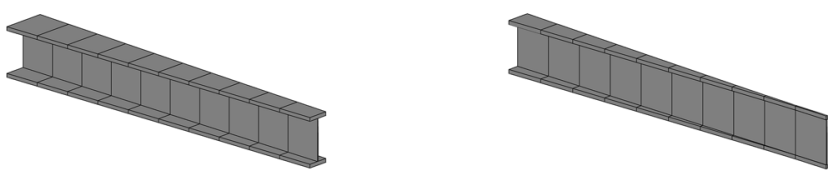

(M2)
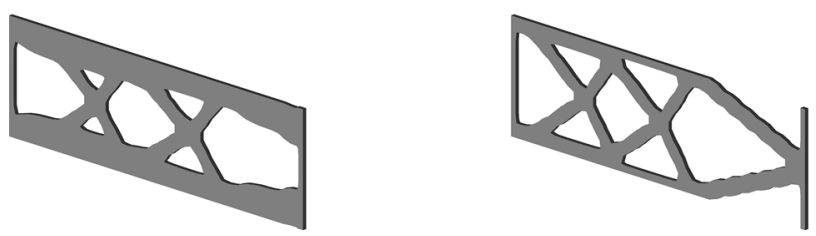

(M3)

Fig. 9 Post-processing results of the optimized component designs of the component models (M1)-(M3) for approach (A) adding complexity to the problem by, e.g., a higher number of components, multiple load cases with different boundary conditions or a selection of available materials, can greatly increase the cost of a monolithic system optimization. In such a scenario, complete decoupling with separate component optimizations could be less expensive (Krischer et al. 2020). Moreover, the training process must not be carried out for every single system optimization. Meta models with a validity over a range of geometrical quantities such as length, width, and height would enhance the applicability to more complex and therefore more expensive problems with a need for higher training cost only once.

Establishing meta models The generation of sample data for the training process of the meta models is a critical step. Besides the great computational cost, the infeasible regions for highly detailed description of components are often located in small areas that need to be captured within the procedure. While training, even if captured, they might be not considered by the feasibility estimator due to unbalanced training data. Therefore, a sufficiently balanced training data set needs to be ensured.

$\kappa$-representation of $K$ The unique representation of component stiffness matrices via an eigenvalue decomposition helps to enable an efficient sample data generation (see Section 3.1). However, a drawback of the approach is that for eigenvector orientations $\gamma$ close to the lower or upper bound, the respective eigenvectors of the opposite bound resemble each other. Hence, the results of the system optimization in terms of $\boldsymbol{\kappa}$ can sometimes differ significantly in $\gamma$ for similar detailed design vectors $\boldsymbol{x}$ (see Section 5.3). Also, since the sample data is not directly derived with respect to $\kappa$, but in terms of the entries of the component stiffness matrix $\boldsymbol{K}$, see (13), the accuracy of the component optimization can only indirectly be ensured by setting a low threshold $\epsilon$. Especially during the sampling procedure, a compromise between a high accuracy and the computational effort must be made.

Post-processing Finally, structural optimization results often need a post-processing step. In Fig. 9, a manual post-processing was carried out. However, small changes might cause a invalid component behavior violating system requirements. Hence, a requirement-based post-processing is necessary to transfer the results into a feasible component.

\section{Conclusion and future work}

The proposed approach in this paper has two main ingredients: (a) a top-down design procedure and (b) establishing 
meta models for feasibility and mass estimation. The topdown design procedure is based on two subsequent optimization problems. First, a system optimization is carried out, that decomposes the problem horizontally and vertically into completely independent component optimization problems. The elastic properties of the mechanical components are hereby defined by component stiffness matrices, which are parametrized by eigenvalues and eigenvectors, enabling better access to the space of stiffness matrices. To assess feasibility and mass of the given component stiffness matrices, meta models are utilized. Feasibility is taken into account by considering geometrical restrictions and material properties. In order to distribute the requirements in a mass-optimal way, a mass estimator is established that provides the lowest possible mass for a given stiffness. As a result of the system optimization, required stiffness properties for both components are assigned, while not knowing their detailed descriptions. In a second step, a subsequent component optimization is using the stiffness properties as constraints. The component optimizations can be carried out completely independently of each other, making any coordination between the components or the system level unnecessary.

For a linear mechanical two-component system, the proposed approach (A) was compared to two alternative design approaches. The approach (B) is a monolithic system optimization, for designing an entire system at once. The uninformed approach (C) distributes a predefined stiffness budget to the components without knowing the effect on mass and feasibility on the component-detail level. Afterwards, two independent component optimization problems are solved with respect to the assigned stiffness budgets. Three different component models were tested: (M1) a i-beam with constant cross-section, (M2) a i-beam with varying flange width, and (M3) an arbitrary body represented by a 2-dimensional topology. It could be shown that the proposed approach (A) is capable of assigning component requirements in a feasible and mass-optimal manner, resulting in designs that only slightly differ from the results of the monolithic optimization of approach (B). The uninformed approaches (C) exhibited problems, in either producing non-feasible requirements (C.1) and/or requirements that lead to suboptimal designs (C.2).

In the future, the approach will be enhanced to problems with (a) multiple interfaces and (b) more degrees of freedom per interface. In order to widen the applicability of meta models, varying geometrical boundary conditions will be considered during the training phase. To reduce the computational effort of the training phase, active-learning sample procedures are to be investigated.

\section{Appendix A}

\section{A.1 Eigenvector derivation}

The eigenvectors $\varphi_{3}$ and $\varphi_{4}$ solved with respect to $l, d_{r}$ and $\gamma$

$$
\begin{aligned}
& \boldsymbol{\varphi}_{3}=\left[\begin{array}{c}
d_{r}^{2} l \frac{\gamma-\left(\frac{-4 d_{r}^{2} \gamma^{2}+2 d_{r}^{2}-l^{2} \gamma^{2}+l^{2}}{l^{2}}\right)^{\frac{1}{2}}}{2 d_{r}^{2}+l^{2}} \\
\gamma \\
-d_{r}^{2} l \frac{\gamma-\left(\frac{-4 d_{r}^{2} \gamma^{2}+2 d_{r}^{2}-l^{2} \gamma^{2}+l^{2}}{l^{2}}\right)^{\frac{1}{2}}}{2 d_{r}^{2}+l^{2}} \\
\frac{-2 d_{r}^{2} \gamma-l^{2}\left(\frac{-4 d_{r}^{2} \gamma^{2}+2 d_{r}^{2}-l^{2} \gamma^{2}+l^{2}}{l^{2}}\right)^{\frac{1}{2}}}{2 d_{r}^{2}+l^{2}}
\end{array}\right] \\
& \boldsymbol{\varphi}_{4}=\left[\begin{array}{l}
\varphi_{41} \\
\varphi_{42} \\
\varphi_{43} \\
\varphi_{44}
\end{array}\right]
\end{aligned}
$$

$$
\begin{aligned}
\varphi_{41}= & -d_{r}^{2} l \frac{\left(\left(4 d_{r}^{2}+l^{2}\right)\left(-4 d_{r}^{2} \gamma^{2}+2 d_{r}^{2}-l^{2} \gamma^{2}+l^{2}\right)\right)^{\frac{1}{2}}+\ldots}{1} \ldots \\
& \ldots \frac{4 d_{r}^{2}\left(\frac{\gamma^{2}\left(4 d_{r}^{2}+l^{2}\right)}{l^{2}}\right)^{\frac{1}{2}}+l^{2}\left(\frac{\gamma^{2}\left(4 d_{r}^{2}+l^{2}\right)}{l^{2}}\right)^{\frac{1}{2}}}{\left(2 d_{r}^{2}+l^{2}\right)\left(4 d_{r}^{2}+l^{2}\right)}
\end{aligned}
$$$$
\varphi_{42}=\frac{-\left(\left(4 d_{r}^{2}+l^{2}\right)\left(-4 d_{r}^{2} \gamma^{2}+2 d_{r}^{2}-l^{2} \gamma^{2}+l^{2}\right)\right)^{\frac{1}{2}}}{4 d_{r}^{2}+l^{2}}
$$

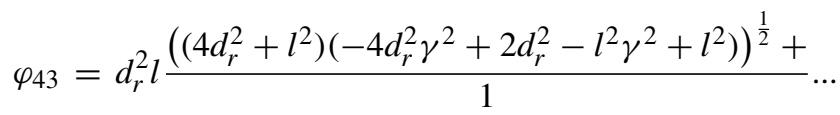

$$
\ldots \frac{4 d_{r}^{2}\left(\frac{\gamma^{2}\left(4 d_{r}^{2}+l^{2}\right)}{l^{2}}\right)^{\frac{1}{2}}+l^{2}\left(\frac{\gamma^{2}\left(4 d_{r}^{2}+l^{2}\right)}{l^{2}}\right)^{\frac{1}{2}}}{\left(2 d_{r}^{2}+l^{2}\right)\left(4 d_{r}^{2}+l^{2}\right)}
$$

$$
\begin{aligned}
\varphi_{44}= & \frac{2 d_{r}^{2}\left(\left(4 d_{r}^{2}+l^{2}\right)\left(-4 d_{r}^{2} \gamma^{2}+2 d_{r}^{2}-l^{2} \gamma^{2}+l^{2}\right)\right)^{\frac{1}{2}}-\ldots}{1} \ldots \\
& \ldots \frac{4 d_{r}^{2} l^{2}\left(\frac{\gamma^{2}\left(4 d_{r}^{2}+l^{2}\right)}{l^{2}}\right)^{\frac{1}{2}}-l^{4}\left(\frac{\gamma^{2}\left(4 d_{r}^{2}+l^{2}\right)}{l^{2}}\right)^{\frac{1}{2}}}{\left(2 d_{r}^{2}+l^{2}\right)\left(4 d_{r}^{2}+l^{2}\right)}
\end{aligned}
$$

\section{A.2 Rigid body mode condensatio $\mathrm{n}$ of the component stiffness matrix}

The component stiffness matrix $\boldsymbol{K}$ can be reduced by taking the rigid body modes $\varphi_{1}$ and $\varphi_{2}$ into account. The resulting linear system of equations

$$
\begin{aligned}
& \boldsymbol{K} \boldsymbol{\varphi}_{1}=0, \\
& \boldsymbol{K} \boldsymbol{\varphi}_{2}=0 .
\end{aligned}
$$


can be solved in terms of the three diagonal terms $K_{11}, K_{22}$ and $K_{44}$ :

$K_{21}=\frac{K_{11} l^{2}+K_{22}-K_{44}}{2 l}$,

$K_{31}=-K_{11}$,

$K_{41}=\frac{K_{11} l^{2}-K_{22}+K_{44}}{2 l}$,

$K_{32}=-\frac{K_{11} l^{2}+K_{22}-K_{44}}{2 l}$,

$K_{42}=\frac{K_{11} l^{2}-K_{22}-K_{44}}{2}$,

$K_{33}=K_{11}$,

$K_{43}=-\frac{K_{11} l^{2}-K_{22}+K_{44}}{2 l}$.

Funding Open Access funding enabled and organized by Projekt DEAL.

\section{Declarations}

Conflict of interest The authors declare that they have no conflict of interest.

Replication of results The results presented in this paper can be completely replicated with MATLAB and the detailed derivations within Sections 3, 4, and 5. The component optimization is based on the 99 line MATLAB topology optimization code of Sigmund (2001) incorporating the Method of Moving Asymptotes (Svanberg 1987). All datasets and codes used in this work are available upon request to the corresponding authors.

Open Access This article is licensed under a Creative Commons Attribution 4.0 International License, which permits use, sharing, adaptation, distribution and reproduction in any medium or format, as long as you give appropriate credit to the original author(s) and the source, provide a link to the Creative Commons licence, and indicate if changes were made. The images or other third party material in this article are included in the article's Creative Commons licence, unless indicated otherwise in a credit line to the material. If material is not included in the article's Creative Commons licence and your intended use is not permitted by statutory regulation or exceeds the permitted use, you will need to obtain permission directly from the copyright holder. To view a copy of this licence, visit http://creativecommons. org/licenses/by/4.0/.

\section{References}

Bathe K-J (2006) Finite element procedures Bathe. Mass, Boston

Bendsøe MP, Sigmund O (2004) Topology optimization: theory, methods and applications, 2nd edn. Springer, Berlin. corrected printing edition

Bishop CM (2009) Pattern recognition and machine learning. Information science and statistics, Springer, New York. corrected at 8th printing 2009 edition

Braun R, Gage P, Kroo I, Sobieski I (1996) Implementation and performance issues in collaborative optimization. 6th Symposium on Multidisciplinary Analysis and Optimization
Cortes C, Vapnik V (1995) Support-vector networks. Mach Learn 20(3):273-297

Ding M, Vemur RI (2005) An active learning scheme using support vector machines for analog circuit feasibility classification. In: Proceedings of the 18th international conference on VLSI Design, pp 528-534

ElMaraghy W, ElMaraghy H, Tomiyama T, Monostori L (2012) Complexity in engineering design and manufacturing. CIRP Ann 61(2):793-814

Guyan RJ (1965) Reduction of stiffness and mass matrices. AIAA J 3(2):380

Hornik K, Stinchcombe M, White H (1989) Multilayer feedforward networks are universal approximators. Neural Netw 2(5):359-366

Huang S, Schimmels JM (1998) Achieving an arbitrary spatial stiffness with springs connected in parallel. J Mech Des 120(4):520-526

Huang S, Schimmels JM (2000) The eigenscrew decomposition of spatial stiffness matrices. IEEE Trans Robot Autom 16(2):146156

Jeong S-H, Choi D-H, Jeong M (2012) Feasibility classification of new design points using support vector machine trained by reduced dataset. Int J Precis Eng Manuf 13(5):739-746

Jung J, Yoon JI, Park S-J, Kang J-Y, Kim GL, Song YH, Park ST, Oh KW, Kim HS (2019) Modelling feasibility constraints for materials design: Application to inverse crystallographic texture problem. Comput Mater Sci 156:361-367

Kim BJ, Yun DK, Lee SH, Jang G-W (2016) Topology optimization of industrial robots for system-level stiffness maximization by using part-level metamodels. Struct Multidiscip Optim 54(4):1061-1071

Kim HM, Michelena NF, Papalambros PY, Jiang T (2003) Target cascading in optimal system design. J Mech Des 125(3):474-480

Kollmann HT, Abueidda DW, Koric S, Guleryuz E, Sobh NA (2020) Deep learning for topology optimization of $2 \mathrm{~d}$ metamaterials. Mater Des 196(4):109098

Krischer L, Sureshbabu AV, Zimmermann M (2020) Modular topology optimization of a humanoid arm: 2020 3rd international conference on control and robots (iccr)

Lei X, Liu C, Du Z, Zhang W, Guo X (2019) Machine learningdriven real-time topology optimization under moving morphable component-based framework. J Appl Mech 86(1):1

Liu G-R, Quek SS (2013) The finite element method: a practical course, 2nd edn. Butterworth-Heinemann, Oxford

Martins JRRA, Lambe AB (2013) Multidisciplinary design optimization: a survey of architectures. AIAA J 51(9):2049-2075

McKay MD, Beckman RJ, Conover WJ (1979) A comparison of three methods for selecting values of input variables in the analysis of output from a computer code. Technometrics 21(2):239

O'Connell RF, Hassig HJ, Radovcich NA (1976) Derivatives of a statically reduced stiffness matrix with respect tosizing variables. J Aircr 13(1):59-60

Oh S, Jung Y, Kim S, Lee I, Kang N (2019) Deep generative design: Integration of topology optimization and generative models. J Mech Des 141(11):436

Shin M-K, Park G-J (2005) Multidisciplinary design optimization based on independent subspaces. Int J Numer Methods Eng 64(5):599-617

Sigmund O (2001) A 99 line topology optimization code written in matlab. Struct Multidiscip Optim 21(2):120-127

Svanberg K (1987) The method of moving asymptotes-a new method for structural optimization. Int J Numer Methods Eng 24(2):359373

Ulu E, Zhang R, Kara LB (2015) A data-driven investigation and estimation of optimal topologies under variable loading configurations. Comput Methods Biomechan Biomed Eng Imag Visual 4(2):61-72 
Viana FAC, Simpson TW, Balabanov V, Toropov V (2014) Special section on multidisciplinary design optimization: Metamodeling in multidisciplinary design optimization: How far have we really come? AIAA J 52(4):670-690

Wang L, Tao S, Zhu P, Chen W (2021) Data-driven topology optimization with multiclass microstructures using latent variable gaussian process. J Mech Des 143(3):296

Wang X, Zhang D, Zhao C, Zhang P, Zhang Y, Cai Y (2019) Optimal design of lightweight serial robots by integrating topology optimization and parametric system optimization. Mech Mach Theory 132:48-65

Wu Z, Fan F, Xiao R, Yu L (2020) The substructuring-based topology optimization for maximizing the first eigenvalue of hierarchical lattice structure. Int J Numer Methods Eng 121(13):2964-2978
Wu Z, Xia L, Wang S, Shi T (2019) Topology optimization of hierarchical lattice structures with substructuring. Comput Methods Appl Mech Eng 345(2):602-617

Yu Y, Hur T, Jung J, Jang IG (2019) Deep learning for determining a near-optimal topological design without any iteration. Struct Multidiscip Optim 59(3):787-799

Zimmermann M, Königs S, Niemeyer C, Fender J, Zeherbauer C, Vitale R, Wahle M (2017) On the design of large systems subject to uncertainty. J Eng Des 28(4):233-254

Zimmermann M, von Hoessle JE (2013) Computing solution spaces for robust design. Int J Numer Methods Eng 94(3):290-307

Publisher's note Springer Nature remains neutral with regard to jurisdictional claims in published maps and institutional affiliations. 